\title{
Loneliness and negative effects on mental health as trade-offs of the policy response to COVID-19
}

\author{
Elena $\operatorname{Popa}^{1}$ (D)
}

Received: 19 August 2020 / Accepted: 18 January 2021 / Published online: 2 February 2021

(C) Springer Nature Switzerland AG 2021

\begin{abstract}
This note introduces a framework incorporating multiple sources of evidence into the response to COVID-19 to overcome the neglect of social and psychological causes of illness. By using the example of psychological research on loneliness and its effects on physical and mental health with particular focus on aging and disability, I seek to open further inquiry into how relevant psychological and social aspects of health can be addressed at policy level.
\end{abstract}

Keywords Loneliness $\cdot$ Mental health $\cdot$ Science and policy

This paper discusses the neglect of mental health and illnesses caused by social isolation in the context of the public health response to COVID-19. ${ }^{1}$ By investigating the example of loneliness, I seek to open a discussion on social and psychological effects brought about by measures such as lockdowns and social distancing as well as on the effectiveness of communication technology in this context. I use the term 'loneliness' in the sense of a subjective experience that may become chronic, affecting both mental and physical health (Cacioppo and Patrick 2008: 5). This investigation will help counter previous tendencies of public health approaches to prioritize biomedical interventions while overlooking psychological and social aspects of health. While fields such as public health or economics have emphasized the conflict between preventing COVID-19 and adverse health effects of lockdowns, or the tension between public health measures and economic activity, I draw from

1 This note belongs to the Topical Collection "Seeing Clearly Through COVID-19: Current and future questions for the history and philosophy of the life sciences", edited by G. Boniolo and L. Onaga.

Elena Popa

elena.popa@auw.edu.bd; elena.popa@protonmail.com

1 Asian University for Women, 20 M.M. Ali Road, Chittagong, Bangladesh 
the philosophy of science. In line with debates on science and values, the proposed framework involves the value described by Longino (1995) as the diffusion of power: accepting multiple disciplinary perspectives as opposed to a dominant approach subsuming the others.

This viewpoint would move beyond biological aspects, seeking available scientific evidence about the trade-offs of lockdowns and social distancing. In this sense, relevant questions include how insights from areas such as social psychology or mental health can help avert effects on mental and physical health and how this can be approached at policy level. I illustrate the proposed framework by looking at how research on loneliness could inform public health policies with the hope of initiating further discussion inclusive of multiple perspectives.

The initial response to COVID-19 seems to have overlooked the possibility of a more nuanced perspective regarding the psychological and social aspects of the pandemic, and loneliness in particular, as potential trade-offs. The World Health Organization (WHO) recommendations on mental health from March 2020 show concern about sustaining social networks: 'if health authorities have recommended limiting your physical social contact to contain the outbreak, you can stay connected via telephone, e-mail, social media or video conference' (WHO 2020: 5). As lockdowns were being enforced, several mental health warnings were issued, particularly regarding vulnerable groups, and recommendations of online therapy were made (Sanchez Nicolas 2020). Subsequent studies called for research on COVID-19 and mental health noting that 'tracking loneliness and intervening early are important priorities' (Holmes et al. 2020: 548). This initial pandemic response suggests that lockdowns and social distancing were taken as central measures, leaving social interaction to technological means. While loneliness and mental health are subsequently highlighted, no reference is made to psychological mechanisms involved in experiencing social isolation, how they affect physical and mental health, or ways of managing them. This raises worries regarding the previous marginalization of mental health among public health measures and the neglect of social causes of illness (Sturgeon 2006; Underwood et al. 1986). ${ }^{2}$

The full anticipation of the impact of lockdowns and social distancing would have been made possible if planners had proactively examined previous research on loneliness. While loneliness is an obvious issue for people living alone, people in joint households constrained to cut their wider social connections are also affected. The distinction between social isolation and loneliness helps explain how these cases are analogous: social isolation is the objective lack of social contact, while loneliness is the subjective feeling of lacking such contacts (Cacioppo and Patrick 2008). The pandemic measures can lead to both social isolation and loneliness, the latter holding for individuals having to cut meaningful interactions outside of their immediate surroundings and feeling that their social needs are not met. The effects are likely to be exacerbated for the older population who was already more likely

\footnotetext{
2 Regarding the latter, as unmet social needs have consequences on physical health, loneliness due to lockdown can be counted as a health risk.
} 
to struggle with loneliness before the pandemic (Courtin and Knapp 2017). ${ }^{3}$ The vulnerability to COVID-19 alongside the alienation by public discourse prioritizing groups perceived as more economically active has placed an additional burden on the elderly (Carrieri et al. 2020). Individuals living with disability form another vulnerable group, also already at higher risk for loneliness and social isolation before the pandemic. Quantitative research has shown that the connection between loneliness and disability is not merely due to medical aspects, but because of structural barriers that prevent disabled individuals from accessing leisure activities outside the home, social interaction, or employment (Macdonald et al. 2018). Approaches incorporating first person perspectives on the experience of disability are particularly important for understanding the link between loneliness and disability. Testimonies on how deaf individuals have been denied both social interaction and the communication of basic needs in institutional setting would be one example (Wong 2020: 59-63). Another example is the phenomenon of shrinking social circles not only for disabled individuals, but also for their family members or friends. This can be explained within a relational model that analyzes disability as experienced through relationships in addition to its medical and social aspects (Kafer 2013: 8). These approaches would be particularly helpful for highlighting how disabled individuals are likely to experience loneliness during the pandemic, and for pointing out potential exacerbating factors.

While loneliness has been acknowledged as a potential issue, it is still unclear how it will be addressed in public health measures. Informing the public about mental and physical effects of loneliness and sketching out ways of coping would be a starting point, given the preexisting research on this. A review article notes that individuals reporting that they frequently feel lonely are at increased risk of cardiovascular disease and accelerated aging, while also being more likely to experience psychotic symptoms, depression, and cognitive decline (Hawkley and Cacioppo 2010). This happens because isolation leads people to feel unsafe and screen their social surroundings for potential threats, being in a constant state of vigilance which leads to the deterioration of social connections and to specific physiological changes. The mechanisms by which loneliness affects mental and physical health have been insufficiently discussed in the context of COVID-19. One relevant instance, with space for more examples to be disclosed by psychology and mental health scholars, is the spiraling trajectory of loneliness. When experiencing loneliness for a prolonged period, one's attempts at socializing are accompanied by defensiveness, which increases the likelihood of rejection, ultimately causing more loneliness (Cacioppo and Patrick 2008: 15-16). This may already be familiar to people stuck with the same household members for a long time, or to people experiencing difficulty socializing after a period of solitude. Again, both social isolation and loneliness can be at play here: an individual whose social needs are not met can feel lonely even in the presence of

\footnotetext{
3 Aspects such as gender, race, or class can also be explored, especially in the light of the previously documented prevalence of mental illness (Rosenfield and Mouzon 2013). As no clear pattern of differences in likelihood of experiencing loneliness has been singled out, I am not discussing them here.
} 
other people and this feeling will cause defensiveness and the subsequent degradation of social connections. Awareness that prolonged isolation takes this form may help a better management of relationships, or lead to seeking psychological help to improve mental well-being. Of acute importance is the case of vulnerable groups such as the elderly and the disabled where the preoccupation for protecting against exposure to the virus needs to be supplemented by attention to social needs in order to counter mental and physical health effects of loneliness.

Another point of potential debate concerns the question whether technology can fully help address mental health needs: while this is recommended by the above mentioned WHO documents, there is room for discussion involving perspectives from psychology, social science, or science and technology studies. Face-to-face emotional support has been shown to be more efficient in preventing depression than support via social media (Shensa et al. 2020). This suggests that policies should also take into account the limitations of communication technology and urge caution about what can be expected from online socialization, also considering the possibility of an increase in levels of loneliness and social isolation. Differences among groups should also be taken into account: if older individuals are at higher risk but also less technologically savvy to what extent can one hope to substitute face-to-face communication? Further research on this is needed with special reference to the COVID-19 context. Transparency about the limitations of technology use can help anticipate or counter their potential drawbacks. Likewise, in the case of disability, acknowledging preexisting structural barriers to meaningful social lives, and taking into account how accessible various means of communication are should inform public health interventions.

As the proposed approach advocates for involving psychologists, mental health professionals, or social scientists in public communication regarding social distancing and mental well-being, it has the advantage of easing the burden for epidemiologists and virologists. Using a plurality of perspectives would fit the broad goal of prevention, while also including the mitigation of public health risks associated with measures such as lockdowns and social distancing.

To conclude, a more nuanced and pluralistic framework bringing together different strands of scholarship can counter the relegation of mental well-being to a secondary role in public health measures and the neglect the social determinants of illness noted in the early public health response to COVID-19. The discussion of loneliness, particularly highlighting the situation of the elderly and the disabled, provides scope for further research and deliberation on policy making in the context of COVID-19 and other health risks requiring analogous measures. Given that the pandemic has brought these issues into the spotlight, policies could rely on existing research to improve the condition of individuals at high risk of developing illnesses because of loneliness.

Acknowledgements I would like to thank two anonymous referees, James Plumtree, Maria Temmes, and Baldur Sigurðsson for their feedback. 


\section{References}

Cacioppo, J. T., \& Patrick, W. (2008). Loneliness: Human nature and the need for social connection. New York: WW Norton \& Company.

Carrieri, D., Peccatori, F. A., \& Boniolo, G. (2020). COVID-19: A plea to protect the older population. International Journal for Equity in Health, 19(1), 1-4.

Courtin, E., \& Knapp, M. (2017). Social isolation, loneliness and health in old age: A scoping review. Health \& Social Care in the Community, 25(3), 799-812.

Hawkley, L. C., \& Cacioppo, J. T. (2010). Loneliness matters: A theoretical and empirical review of consequences and mechanisms. Annals of Behavioral Medicine, 40(2), 218-227.

Holmes, E. A., O’Connor, R. C., Perry, V. H., Tracey, I., Wessely, S., Arseneault, L., et al. (2020). Multidisciplinary research priorities for the COVID-19 pandemic: A call for action for mental health science. The Lancet Psychiatry., 7, 547-560.

Kafer, A. (2013). Feminist, queer, crip. Bloomington: Indiana University Press.

Longino, H. E. (1995). Gender, politics, and the theoretical virtues. Synthese, 104(3), 383-397.

Macdonald, S. J., Deacon, L., Nixon, J., Akintola, A., Gillingham, A., Kent, J., et al. (2018). 'The invisible enemy': Disability, loneliness and isolation. Disability \& Society, 33(7), 1138-1159.

Rosenfield, S., \& Mouzon, D. (2013). Gender and mental health. In C. S. Aneshensel, J. C. Phelan, \& A. Bierman (Eds.), Handbook of the sociology of mental health (pp. 277-296). Dordrecht: Springer.

Sanchez Nicolas, E. (2020). WHO warning on lockdown mental health. https://euobserver.com/coron avirus/147903.

Shensa, A., Sidani, J. E., Escobar-Viera, C. G., Switzer, G. E., Primack, B. A., \& Choukas-Bradley, S. (2020). Emotional support from social media and face-to-face relationships: Associations with depression risk among young adults. Journal of Affective Disorders, 260, 38-44.

Sturgeon, S. (2006). Promoting mental health as an essential aspect of health promotion. Health Promotion International, 21(suppl_1), 36-41.

Underwood, P., Owen, A., \& Winkler, R. (1986). Replacing the clockwork model of medicine. Community Health Studies, 10(3), 275-283.

WHO. (2020). Mental health and psychosocial considerations during the COVID-19 outbreak. https:// www.who.int/docs/default-source/coronaviruse/mental-health-considerations.pdf.

Wong, A. (Ed.). (2020). Disability visibility: First-person stories from the twenty-first century. New York: Vintage.

Publisher's Note Springer Nature remains neutral with regard to jurisdictional claims in published maps and institutional affiliations. 